\title{
A technical soda lignin as sole carbon-diet for a higher termite species, Nasutitermes ephratae: insight into the changes in the gut bacterial community and in the lignin phenolic fraction
}

Edouard Miambi ( $\nabla$ miambi@u-pec.fr )

Universite Paris-Est Creteil Val de Marne

My Dung Jusselme

Universite Paris-Est Creteil Val de Marne

Laurent Cézard

INRA Centre de Versailles-Grignon

Florian Pion

INRA Centre de Versailles-Grignon

Stéphanie Baumberger

INRA Centre de Versailles-Grignon

Alain Robert

INRA Centre de Versailles-Grignon

Catherine Lapierre

INRA Centre de Versailles-Grignon

Michel Diouf

Universite Paris-Est Creteil Val de Marne Institut d'urbanisme de Paris

Philippe Mora

Universite Paris-Est Creteil Val de Marne

Research article

Keywords: Technical lignin, Protobind 1000, grass soda lignin, natural biomass utilization systems (NBUS), gut bacterial community, higher termite, Py-GC/MS, biorefinery

Posted Date: May 26th, 2020

DOI: https://doi.org/10.21203/rs.3.rs-28014/v1

License: (9) This work is licensed under a Creative Commons Attribution 4.0 International License. Read Full License 

1 A technical soda lignin as sole carbon-diet for a higher termite species, Nasutitermes ephratae:

2 insight into the changes in the gut bacterial community and in the lignin phenolic fraction

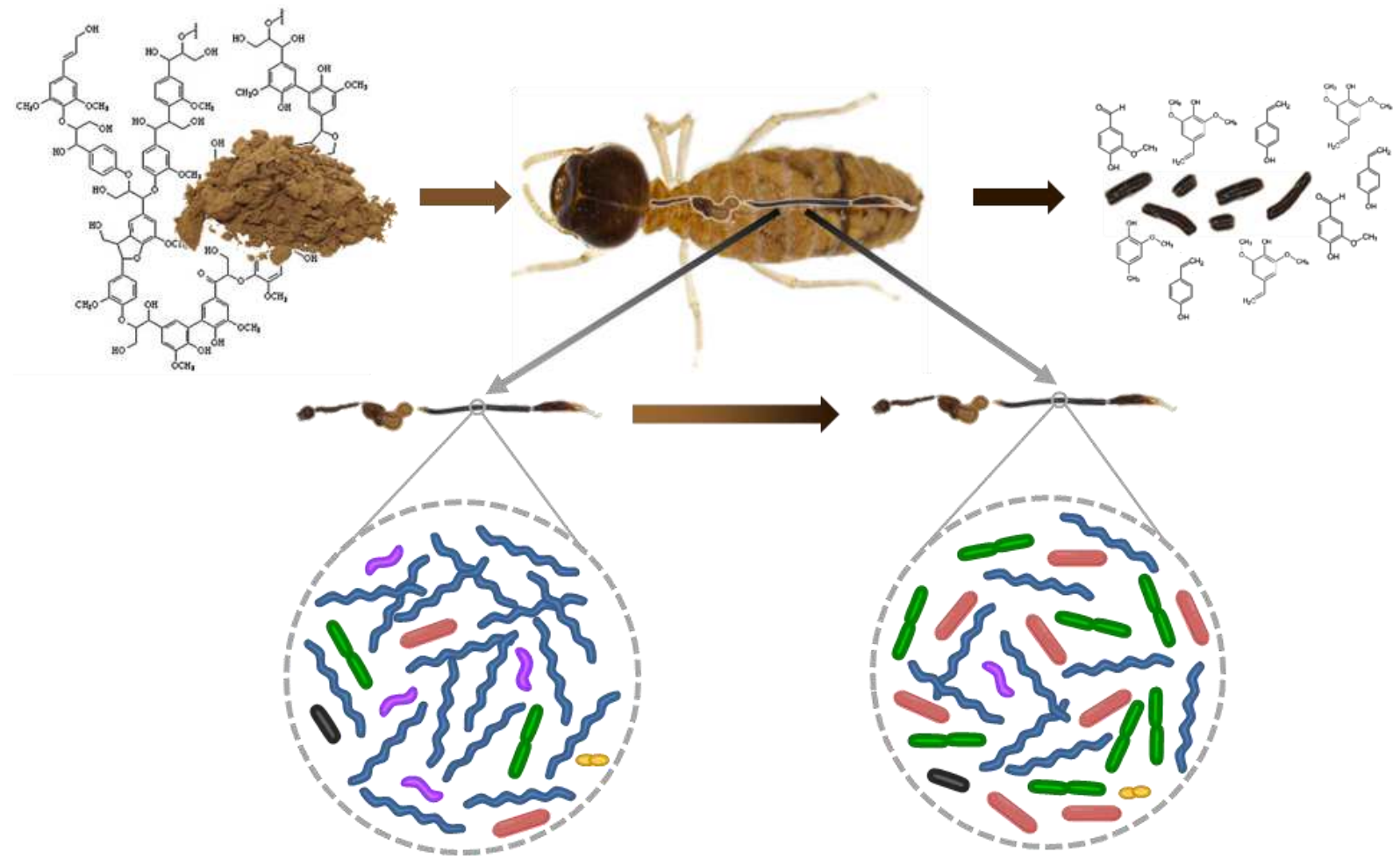

My Dung JUSSELME ${ }^{\mathrm{a}}$, Laurent Cézard ${ }^{\mathrm{b}}$, Florian Pion ${ }^{\mathrm{b}}$, Stéphanie Baumberger ${ }^{\mathrm{b}}$, Alain Robert ${ }^{\mathrm{a}}$, Catherine Lapierre $^{b^{*}}$, Michel Dioufa, Philippe Mora ${ }^{a}$, Edouard Miambia*

Institut Jean-Pierre Bourgin, UMR1318 INRA-AgroParisTech-ERL3559 CNRS, 78026 Versailles Cedex France

Institut d'écologie et des sciences de l'environnement de Paris (iEES Paris), Université Paris Est Créteil, 94014 Créteil Cedex France

*Corresponding authors: miambi@u-pec.fr;

Université Paris Est Créteil, Institut d'Ecologie et des Sciences de l'Environnement de Paris (iEES Paris), 94014 Créteil Cedex France 


\section{Abstract}

\section{Background}

Termites account for natural biomass utilization systems (NBUS) that evolved the ability to overcome the overall recalcitrance of lignins towards lignocellulose transformation processes. With the objective of applying this capacity to the conversion of technical lignins produced by biorefineries, a higher wood-feeding termite species, Nasutitermes ephratae was fed with a commercial grass soda lignin (Protobind 1000, PB1000). The survival rates of Protobind 1000-fed termites were determined as well as changes in the structure of gut bacterial community and in the chemical composition of this technical lignin.

\section{Results}

The ingestion of PB1000 by worker castes of $N$. ephratae was revealed by Pyrolysis-Gas Chromatography Mass Spectrometry (Py-GC/MS) analyses directly performed on termites. Survival rates were reduced by two -fold in the termites fed with PB1000 compared to controls. The relative abundance of Firmicutes and Bacteroidetes increased in the gut bacterial community of termites fed with PB1000. The digestion of PB1000 by termites triggered an increase in the syringyl-to-guaiacyl (S/G) ratios. These changes in the chemical composition of PB1000 in the gut of termites was marked by a decrease in relative content of free phenolic monomers.

\section{Conclusion}

This work showed the abilities of digestive tract of a wood-feeding higher termite species, N. ephratae to metabolize the fraction of the volatile phenolic monomers of PB1000. Overall, our results provide insights into the bacterial lineage candidates for development of bacterial inoculum for pretreatment processes in valorization of technical lignin in biorefinery.

Keywords: Technical lignin, Protobind 1000, grass soda lignin, natural biomass utilization systems (NBUS), gut bacterial community, higher termite, Py-GC/MS, biorefinery 


\section{INTRODUCTION}

The recalcitrance of lignins towards physicochemical and biochemical treatments is an obstacle to using lignocellulosic biomass as raw material for the production of biofuels and value-added biochemicals $[\underline{1}, \underline{2}]$. Consequently, over the last decades, studies have been directed towards Natural Biomass Utilization Systems (NBUS) which refers to natural systems that can efficiently degrade and utilize lignocellulosic biomass []ㅡ. Termites consume 3-7 billion tons of lignocellulosic materials annually [4] and thereby represent one of the most prolific and efficient NBUS. By virtue of their microbial symbionts, which mostly consist in novel lineages that have co-evolved or converged with their specific host []ㅡ, termites developed efficient mechanisms of biomass utilization. These microbial communities are very dense (up to $10^{11}$ cells $/ \mathrm{mL}$ ), diverse $(6,000$ phylotypes $/ \mathrm{mL}$ or 740 phylotypes by gut), corresponding to many lineages of mostly uncultivated bacteria that exclusively occur in this habitat [6]. Many putative cellulases, xylanases and other glycoside hydrolases assigned to the symbiont bacterial groups were encountered in termite-guts and are thought to be involved in lignocellulose degradation [7]. Lignocellulose digestion in termites is accomplished by a dual system combining activities of both the host and its bacterial symbionts [8].

Extensive work has been devoted to study lignocellulose degradation by wood-feeding species of socalled lower termites. Lower termites harbor symbiotic flagellate protozoa known to be sources of cellulases and hemicellulases. Evidence has been brought of the abilities of their digestive system and the associated gut bacteria to metabolize aromatic compounds and dimeric lignin models [9-11]. However, most previous studies devoted to the in-vivo degradation of the aromatic polymer lignin by insects also focused on lower wood-feeding termites. Modifications of lignin in the digestive tract were found to be either weak $[\underline{12}, \underline{13}]$ or more pronounced [14-17]. These discrepancies may be accounted for by the structural variability of lignins used in these studies (according to their botanical origin and/or their isolation procedure) and by the employed analytical tools.

Fewer studies have been devoted to lignin degradation by higher termites which harbor exclusively prokaryotic symbionts and display dietary diversification $[\underline{18}, \underline{19}]$. The loss of flagellates in higher 
termites suggests the gain of new functions by the prokaryotic microorganisms in the digestive process [19]. Many lignin degrading candidates are reported, but single strain shows limited lignin utilization ability [20]. With the emerging role for bacteria in lignin degradation [21, 22], the exploration of the greater bacterial biodiversity in the gut of higher termites as bacterial catalytic systems could provide an insight into the development of inoculum for pretreatment processes in valorization of technical lignins.

The present study investigates for the first time, whether a technical grass soda lignin, Protobind 1000 (PB1000) can be metabolized by the gut microbiota of a higher termite species. We have hypothesized that the pronounced axial dynamics of oxygen status and redox potential, together with the

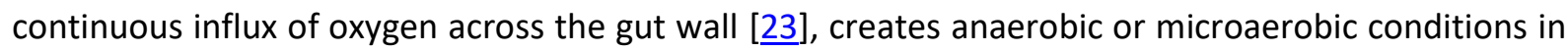
the gut of higher termites. This environment maybe especially interesting as canonical lignin degradation involves peroxide- or oxygen-dependent enzymes [24]. To test this hypothesis, a higher wood-feeding termite species, $N$. ephratae was fed with PB1000. The effects of PB1000 on the physiological activities of $N$. ephratae were investigated by monitoring (i) the survival rates of termite as compared to usual birch wood diet, (ii) the changes in the gut bacterial community (iii) and the modifications of the chemical structure of this technical lignin. To assess the changes in termite-gut bacterial, classification level at phylum and family of bacterial 16S rRNA genes was performed using next generation sequencing method as described in most previous studies on termite gut microbiota

[25, 26]. Analytical pyrolysis combined to gas chromatography/mass spectrometry (Py-GC/MS) is widely used to investigate the chemical structure of lignins and biomass $[\underline{27}, \underline{28}]$. This method was selected in the present study to compare the chemical structure of the undigested PB1000 with digested samples in termite-gut.

\section{RESULTS}

\section{Chemical composition of PB1000 and effect on survival rates of termites}

Klason lignin was the major constituent of the PB1000 sample (Table 1). Neutral sugars accounted for $1.9 \%$ and were mainly composed of xylose ( $67 \%$ of total carbohydrates). The monomeric phenolics 
Table 1: Compositional analysis of the PB1000 sample

accounted for about $1.4 \%$ of the PB1000 sample and the main compounds in decreasing order of abundance were acetosyringone $>$ syringaldehyde $>$ vanillin $>p$-coumaric acid $>$ ferulic acid.

\begin{tabular}{ll}
\hline Composition & Amount $(\mathrm{mg} / \mathrm{g})$ \\
\hline Klason lignin $^{\mathrm{a}}$ & $881.1(4.6)$ \\
\hline $\begin{array}{l}\text { Total carbohydrates }^{\mathrm{b}} \\
\quad \text { including }\end{array}$ & $18.51(0.83)$ \\
$\quad$ xylose & $12.31(0.14)$ \\
$\quad$ arabinose & $2.67(0.02)$ \\
glucose & $2.14(0.49)$ \\
galactose & $1.39(0.21)$ \\
\hline Total phenolic monomers & $13.7(4.6)$ \\
$\quad$ including & \\
acetosyringone & $4.76(0.08)$ \\
syringaldehyde & $2.27(0.04)$ \\
vanillin & $1.99(0.04)$ \\
-Coumaric acid & $1.13(0.01)$ \\
ferulic acid & $1.09(0.03)$ \\
\hline Ash & $14.4(1.99)$ \\
\hline
\end{tabular}

Data are mean values $(n=3)$. In parentheses are the values of the standard deviations

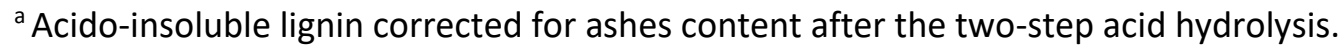

${ }^{\mathrm{b}}$ Neutral monosaccharides determined after TFA hydrolysis (no cellulose-derived glucose detected after severe $\mathrm{H}_{2} \mathrm{SO}_{4}$ hydrolysis).

Figure 1 shows the survival rates of termites fed on PB1000 and birch sawdust used as control. In both cases, survival rates decreased with increasing feeding time. The survival rates of birch-fed termites and PB1000-fed termites were in the same order of magnitude the first day after the beginning of the experiments. From the second day, the decrease of the survival rates was higher in PB1000-fed termites than in birch-fed termites. The differences in survival rates between the two treatments were statistically significant ( $P<0.05$, Fischer's PLSD-test) from the second day until the end of experiments (6 days). 
The gut bacterial community of worker termites determined directly after collection from the nest was dominated by the Spirochaetes followed by Fibrobacteres that accounted for about $67 \%$ and $18 \%$ respectively (Fig.2). The relative abundance of the Spirochaetes decreased when termites were fed with PB1000 as well as with birch wood. The decrease of the relative abundance of members of this phylum was higher in the gut of PB1000-fed termites than in those fed with birch wood. As a consequence, the relative abundance of the Bacteroidetes and Firmicutes increased with a remarkable decrease in members of Fibrobacteres. In termites fed with birch wood, there were no noticeable changes in the structure of bacterial community at 3 and 6 days. Notably, the relative abundance of the Fibrobacteres was of the same order of magnitude in comparison with termites directly collected from the nest.

Analysis at the family level revealed that Spirochaetes were mainly represented by phylotypes assigned to Family Spirochaetaceae_Treponema across all samples (Fig.3). Feeding termites with PB1000 drastically affected members assigned to Termite_cluster_I (Fibrobacteres) and at lower extent those assigned to Rhodospirillaceae (Proteobacteria). In contrast, PB1000-diet triggered an increase in the relative abundance of members of Porphyromonadaceae_3, Porphyromonadaceae_Gut_group, Rikenellaceae (Bacteroidetes); Desulfovibrionaceae (Proteobacteria); Insect_cluster, Family_XIIIIncertae_Sedis, Ruminococcaceae, and Peptococcaceae_1 (Firmicutes).

Py-GC/MS analyses of birch wood and PB1000 before and after digestion in the gut of $N$. ephratae

The Py-GC/MS pyrograms of the major volatile compounds released by pyrolysis of the initial PB1000 and birch wood samples as well as guts from termites fed during 6 days with PB1000 or birch wood samples are shown in Fig.4. The main recovered phenolics were representatives of guaiacyl G compounds (peaks $3,4,6,7,9,11,12$ and 14) and syringyl S compounds (peaks $8,10,13,15,16,17$, 18 and 19). Vinylphenol (peak 5) was observed only in the pyrograms from PB1000. The total of G compounds was calculated without including vinylguaiacol (peak 7) as this product may originate from 
performed from the termite gut samples containing either the digested PB1000 or the digested birch

137 wood sample new peaks labelled P could be seen that could be assigned to protein-derived pyrolysis

138 products (Fig. 4B and 4D).

139 The relative percentages of the main phenolics released from the pyrolysis of birch wood or of PB1000

140 samples, before and after digestion in N. ephratae gut are given in Table 2.

141 Table 2: Relative abundance of the major low molecular weight phenolics released by pyrolysis of birch

142 wood and of PB1000 samples before and after a 6-day long digestion in N. ephratae gut (expressed in

$143 \%$ of their total area). S: syringyl-type phenolic compounds; G: guaïacyl-type phenolic compounds.

\begin{tabular}{|c|c|c|c|c|}
\hline \multirow{2}{*}{$\begin{array}{l}\text { Pyrolysis compounds } \\
\text { (peak number presented in Fig. 4) }\end{array}$} & \multicolumn{2}{|c|}{ Birch wood } & \multicolumn{2}{|c|}{ Protobind 1000} \\
\hline & Initial sample & In termite guts & Initial sample & In termite guts \\
\hline $\begin{array}{c}\text { Vinylphenol (VP) } \\
(\text { peak 5) }\end{array}$ & Traces & Traces & $3.97(0.17) b$ & $3.35(0.18) a$ \\
\hline $\begin{array}{c}\text { Vinylguaiacol (VG) } \\
\text { (Peak 7) } \\
\end{array}$ & $8.97(0.50) a$ & $13.31(0.26) b$ & $13.68(0.85) b$ & $13.25(1.61) b$ \\
\hline $\begin{array}{c}\text { G compounds (sum of peaks } 3,4,6 \text {, } \\
99,11,12,14)\end{array}$ & $22.68(1.24) \mathrm{a}$ & $25.22(0.76) b$ & $30.08(0.22) \mathrm{c}$ & $25.61(0.28) b$ \\
\hline $\begin{array}{l}\text { S compounds (sum of peaks } 8,10, \\
13,15 \text { to } 19)\end{array}$ & $68.34(1.66) d$ & $61.48(0.87) c$ & $52.28(1.12) a$ & $57.80(2.07) b$ \\
\hline S/G ratio & $3.03(0.23) c$ & $2.44(0.11) b$ & $1.74(0.05) a$ & $2.26(0.10) \mathrm{b}$ \\
\hline $\begin{array}{r}\text { Vanillin } \\
(\text { peak 9) }\end{array}$ & $2.35(0.14) b$ & Traces & $1.42(0.03) \mathrm{a}$ & Traces \\
\hline $\begin{array}{l}\text { Syringaldehyde } \\
\text { (peak 16) }\end{array}$ & $4.38(0.32) b$ & Traces & $2.19(0.08) a$ & Traces \\
\hline $\begin{array}{l}\text { Acetosyringone } \\
\text { (peak 18) }\end{array}$ & $3.86(0.48) b$ & $1.72(0.06) a$ & $7.15(0.30) c$ & $1.81(0.07) a$ \\
\hline
\end{tabular}

144 Data are mean values $(n=3)$. In parentheses are the values of the standard deviations. Differences were

145 tested using ANOVA analysis according to Fisher's PLSD post-hoc tests and values overwritten with

146 different letters are statistically significantly different $(P<0.05)$.

147 Digestion in termite guts resulted in an increase of the pyrolysis S/G ratios of PB1000 samples and a

148 decrease of birch samples. The results were significantly different $(P<0.05$, Fisher's PLSD post-hoc

149 tests). The relative abundances of vanillin (G-CHO, peak 9), syringaldehyde (S-CHO, peak 16) and

150 acetosyringone $\left(\mathrm{S}-\mathrm{CO}-\mathrm{CH}_{3}\right.$, peak 18$)$ significantly decreased for both samples $(\mathrm{P}<0.05$, Fisher's PLSD

151 post-hoc tests). 
As the compositional analysis of PB1000 revealed the occurrence of acetosyringone, syringaldehyde and vanillin as the main low molecular weight phenolics of the PB1000 sample (Table 1), the contribution of these free phenolics to the pyrolysis trace was evaluated by subjecting PB1000 to a first thermal treatment at $200^{\circ} \mathrm{C}$ aimed at volatilizing low molecular weight compounds, followed by the pyrolysis step at $500^{\circ} \mathrm{C}$ (See additional file 1). By doing so, we could ascertain that these phenolics released by pyrolysis of the initial PB1000 sample partially originate from free acetosyringone, syringaldehyde and vanillin. These compounds were almost absent from the pyrogram of the termite guts containing the digested PB1000 sample.

\section{DISCUSSION}

There is still little information in literature on the capability of the degradation of technical lignin by higher termites, since most studies have focused on the lower termites and on native lignins. This is the first study on the fate of a technical grass soda lignin, PB1000, in the digestive system of a higher wood-feeding termite species, $N$. ephratae. It also provides information on the effect of ingestion of PB1000 by termites in the survival rates and in the structure of gut-bacterial community. The substrate PB1000 was selected for its commercial availability and for its frequent use in research and development projects [로].

The results obtained in the present study showed that feeding termites with PB1000, a grass soda lignin, lowered the survival rate. Both the low carbohydrate content (mainly derived from hemicelluloses) and the presence of low molecular weight phenolics in PB1000 (such as syringaldehyde, acetosyringone) were susceptible to alter the physiology of the termites and jeopardize their survival. Likely, the decrease in termite survival rates in the presence of PB1000 was due to the low molecular weight phenolics confirming the statements that these compounds are among the mainly low molecular weight extractives which are known to inhibit the biological activities of termites [30]. The lower termite survival rates could also be explained by the repulsive effect of PB1000 observed during experiments, keeping away termites from the substrate. This probably caused 
the termites to die by starvation. Unfortunately, the lack of non-fed control made it impossible to draw

178 any conclusions on this point.

179 However, the differences in gut bacterial community of termite fed with PB1000 compared to the usual birch wood-diet clearly established the ingestion of these two substrates by termites. In line with that of congeneric Nasutitermes spp. $[\underline{7}, \underline{25}, \underline{31}]$, the structure of bacterial gut community in birch woodfed termites (control) showed the predominance of Spirochetes and Fibrobacteres. By contrast, ingestion of PB1000 triggered the decrease of the relative abundance of these phyla and the increase of the Firmicutes and Bacteroidetes. Changes in the structure of the gut bacterial community was expected since it has been well documented that different dietary habits shape the structure of termite-gut microbiota [32-34]. Of a particular importance in the present study is the determination of the bacterial groups which predominate in the community when termites were fed with PB1000. We used NGS approach to characterize the structure of bacterial community. Since the classification of the short sequence reads is limited by the taxonomic depth and resolution [35], in the present study, we used a curated reference database for accurate taxonomic analysis of the bacterial gut microbiota of dictyopteran insects [36]. Interestingly, we found that the predominant lineages were specific termite-gut OTU previously reported [e.g. Ruminococcaceae, and Peptococcaceae_1, Porphyromonadaceae_Gut_Group and Rikenellaceae (Bacteroidetes) [25, 26] . Our findings may be valuable for the development of bacterial inoculum for pretreatment processes in valorization of technical grass soda lignins. Indeed, the predominance of Firmicutes seems to be a general trend in the guts of termites fed with grass-based diets [37]. Interestingly, phylotypes belonging to Firmicutes have been reported to be involved in lignin degradation [22, $\underline{38}, \underline{39}]$, as well as in secondary metabolite detoxification [40]. Bacteroidetes might similarly be echoed by the recently reported lignin degrading potential of Sphingobacterium sp. T2 able to degrade industrial lignins with extracellular manganesedependent superoxide dismutases [41]. But, the increase of the relative abundance in Firmicutes and 
for the growth of phylotypes belonging to these phyla or the death of some phylotypes mainly relatives of Spirochaetes and Fibrobacteres due to the ingestion of PB1000 by termites. Further studies are needed to elucidate this question.

It is worth noting that in the present study, Py-GC/MS was found to be a suitable method for the analysis of the chemical composition of undigested and digested birch wood and PB1000. The PyGC/MS evaluation of digested lignocellulosic substrates can be directly performed within the collected guts, without any isolation or purification step. Pyrograms of Py-GC/MS analyses from termite guts containing the ingested PB1000 or birch wood samples displayed protein-derived products which did not interfere with the $\mathrm{G}$ and $\mathrm{S}$ components.

In agreement with previous studies, the main compounds released by pyrolysis of birch wood and PB1000 were representatives of $G$ and of $S$ compounds $[\underline{42}, \underline{43}]$. The specific release of vinylphenol from PB1000 pyrolysis confirmed the occurrence of $p$-coumaric units in this sample, as free and/or esterified acid. Moreover, the detection of 4-hydroxy-5,6-dihydropyran-2-one (peak 1) was consistent with the presence of some hemicellulose derivatives in PB1000, since this product originates from C5 sugars [42]. Of particular interest were also the observations that the digestion of PB1000 in termite guts induced a strong relative decrease of vanillin, syringaldehyde and acetosyringone in the pyrograms. As these carbonyl compounds initially occurred in the PB1000 sample, this result reveals that they have been eliminated in termite guts, either by action of their microbiota and/or by their degradation into non-volatile and resistant structures.

Changes in the pyrolysis $S / G$ ratio of the substrates after digestion support the idea of their chemical modifications in the digestive tract of $N$. ephratae. Strikingly, while the pyrolysis S/G ratio of PB1000 sample was decreased by gut digestion, the pyrolysis S/G ratio of birch wood was found to be increased. These opposite variations indicate that both substrates were degraded in the guts, but by different mechanisms. That different lignin-containing substrates are differently degraded is in agreement with literature data [2]. In the case of the birch wood sample, the $G$ and S pyrolysis products essentially originate from $\mathrm{G}$ and $\mathrm{S}$ lignin units involved in labile ether bonds. On this basis, the reduction 
of the pyrolysis S/G ratio induced by gut digestion of birch wood suggests that S lignin units involved

230 in $\beta-0-4$ bonds were more degraded than the $\mathrm{G}$ analogous structures. In contrast, as reported for lower

231 termites fed with grass lignocellulose [43], the pyrolysis S/G ratio in PB1000-fed termites increased, suggesting that $\mathrm{G}$ lignin units might be more degraded than $\mathrm{S}$ ones. This preferential degradation of $\mathrm{G}$ units in PB1000 might be related to the fact that most $\mathrm{G}$ units in grass lignins are terminal units with free phenolic groups [44] which make them more susceptible to oxidative degradations. Taking together, our results suggest that the fraction of free low-molecular weight phenolics in PB1000 was metabolized in the digestive system of $N$. ephratae.

\section{CONCLUSION}

Termite feeding experiments and Py-GC/MS analyses of gut contents allowed to provide herein the first demonstration that the technical grass soda lignin PB1000) can be ingested by a higher woodfeeding termite, $N$. ephratae, but with some detrimental impact on the survival rates of termites. The ingestion of PB1000 resulted in changes in the structure of the gut-bacterial community consistently encountered in Nasutitermes spp in favor of Firmicutes and Bacteroidetes. The digestive system of termite species, N. ephratae induced the degradation of some PB1000 aromatic compounds. This was ascertained by Py-GC/MS analyses directly performed on termite guts, without any time-demanding isolation step. Overall, our results pave the way for the development of further strategies aiming at improving the survival rate of termites fed with technical lignins. They also provide insights into bacterial groups that can be used for mimicking termite digestive strategies for biological conversion of biorefinery wastes.

\section{MATERIAL AND METHODS}

\section{Technical lignin origin and composition}

A commercial grass soda lignin, PB1000 (Green Value Entreprises LLC, USA), isolated from mixed wheat straw/sarkanda bagasse, was used for the termite feeding experiments. Lignin was determined as Klason lignin (KL) determination. KL content was determined gravimetrically after a two-step sulfuric acid hydrolysis of the sample (300 mg), with correction for ash content, as described previously [45]. 
Neutral sugars were analyzed by a sequential two-step acidic hydrolysis of the sample $(10 \mathrm{mg})$ with aqueous trifluoroacetic acid (TFA, $\left.2.3 \mathrm{M}, 2 \mathrm{~h}, 110^{\circ} \mathrm{C}\right)$, then sulfuric acid $\left(51 \% \mathrm{w} / \mathrm{w} \mathrm{H}_{2} \mathrm{SO}_{4}, 1 \mathrm{~h}\right.$, ambient temperature; then $5 \% \mathrm{w} / \mathrm{w} \mathrm{H}_{2} \mathrm{SO}_{4}, 2 \mathrm{~h}, 100^{\circ} \mathrm{C}$ ). The neutral monosaccharides recovered after TFA and $\mathrm{H}_{2} \mathrm{SO}_{4}$ hydrolysis were assigned to hemicellulose-derived sugars and cellulose-derived glucose, respectively, and determined by high-performance anion-exchange chromatography with amperometric detection and fucose as internal standard according to Sipponen et al. [46]. The extraction of free phenolics was performed from $10 \mathrm{mg}$ sample put together with $0.025 \mathrm{mg}$ o-coumaric acid (internal standard) in $1 \mathrm{~mL}$ water containing $0.1 \%(\mathrm{v} / \mathrm{v}) \mathrm{HCOOH}$. The extraction was performed for $2 \mathrm{~h}$ at room temperature and with constant agitation at $350 \mathrm{rpm}$. The extraction mixture was then centrifuged at 2,000 $\mathrm{g}$ for $10 \mathrm{~min}$ at room temperature. The supernatant was then purified onto a Sep-Pack tC18 cartridge (Waters, Guyancourt, France) and then analyzed by HPLC-UV, as previously described by Lapierre et al. [47]. All the analyses were performed in duplicates (error $<3 \%$ ). All the compositional values are expressed in weight percent.

\section{Termite collection and feeding experiments}

Higher wood-feeding termites, N. ephratae, were obtained from a laboratory colony at IEES (Institute of Research for Development - France Nord) at Bondy. The colony was maintained in termite rearing room on a 12 -h light/dark cycle at $27^{\circ} \mathrm{C} \pm 2{ }^{\circ} \mathrm{C}, 80 \%$ relative humidity and fed with birch wood. Experiments were implemented with worker termites.

Based on our previous studies on termite behavior and handling, 200 worker castes were placed in a crystal Polystyrene Lab box (L60 X W43 X H50 mm) (Thermo Fisher Scientific, France) containing $20 \mathrm{~g}$ of sterile sand of Fontainebleau mixed with either powdered PB1000 (0.25 g per box), or birch sawdust ( $0.25 \mathrm{~g}$ per box) serving as controls. About $0.5 \mathrm{~mL}$ of sterile distilled water was added every 3 days in each box for humidification of the sand of Fontainebleau. The number of live workers was recorded every day for 6 days. Determination of termite survival was performed in triplicate. At the end of experiments, termite guts were isolated using fine sterile forceps and placed into $2-\mathrm{mL}$ eppendorf 
tube. Termite-guts were immediately frozen at $-20^{\circ} \mathrm{C}$ prior DNA extraction or frozen at $-20^{\circ} \mathrm{C}$ and freeze-dried for Py-GC/MS analysis.

\section{DNA extraction, amplification, sequencing and sequence processing}

Twenty-five termite-guts were first crushed using a polypropylene micro pestle in $1.5 \mathrm{~mL}$ microtube containing $1 \mathrm{~mL}$ of Ringer solution [48] and centrifuged at $11,000 \times \mathrm{g}$ for $15 \mathrm{~min}$ at $4^{\circ} \mathrm{C}$. The pellets were suspended in the NucleoSpin ${ }^{\circ}$ Soil Solution C1 buffer. Then DNA was extracted using the NucleoSpin Soil isolation kit (Macherey-Nagel, Germany) in accordance with manufacturer's instructions. DNA extracts from triplicate were pooled and concentration was quantified using a ND1000 Spectrophotometer (NanoDrop products, Wilmington, USA). DNA aliquots with equal concentrations were sent to the Research and Testing Laboratory (Lubbock, TX, USA) for amplification and sequencing. The V1-V2 variable regions of the $16 \mathrm{~S}$ rRNA genes were amplified using the bacterial primers $28 \mathrm{~F}$ and $338 \mathrm{R}$ and sequenced using $2 \times 250$ paired-end Illumina MiSeq platform. The raw sequence data have been deposited in the NCBI Sequence Read Archive under the BioProject PRJNA550212.

Data were demultiplexed and pair-end reads were joined by the GeT platform, using Flash v1.2.6 [49] and the barcode and primer sequences were removed with cutadapt [무]. Subsequently, the sequences were aligned to the SILVA reference database release 128 [51] and preclustered (pre.cluster, diffs $=1$ ). Chimeras were removed using VSEARCH [52]. To account for differences in sampling effort, 12,559 sequences were randomly subsampled from the dataset. Sequences were then clustered into operational taxonomic unit (OTU) with SWARM [53] which generates the OTU's abundance table with an OTU being defined at the $97 \%$ sequence similarity level. Taxonomic affiliation for each OTU were determined using DictDB v3, the reference database dedicated to insect-associated bacteria [36].

\section{Py-GC/MS analyses}

The initial birch wood sawdust and PB1000 samples as well as the freeze-dried guts of termites fed with either birch or PB1000 samples were subjected to Py-GC/MS analyses as recently described by 
Voxeur et al. [54]. Analyses were carried out using a CDS model 5250 pyroprobe autosampler interfaced to an Agilent 6890/5973 GC/MS. The samples (400 $\mu \mathrm{g}$ ) were pyrolyzed in a quartz tube at $500^{\circ} \mathrm{C}$ for $15 \mathrm{~s}$. The volatile pyrolysis products were separated on a GC capillary column (5\% phenyl methyl siloxane, $30 \mathrm{~m}, 250 \mu \mathrm{m}$ i.d., and $0.25 \mu \mathrm{m}$ film thickness) using helium as the carrier gas with a flow rate of $1 \mathrm{~mL} / \mathrm{min}$. The pyrolysis and GC/MS interfaces were kept at $290^{\circ} \mathrm{C}$ and the $\mathrm{GC}$ was temperature-programmed from $40^{\circ} \mathrm{C}(1 \mathrm{~min})$ to $130^{\circ} \mathrm{C}$ at $+6^{\circ} \mathrm{C} \mathrm{min}-1$, then from 130 to $250^{\circ} \mathrm{C}$ at $+12^{\circ} \mathrm{C}$ $\mathrm{min}^{-1}$ and finally from $250^{\circ} \mathrm{C}$ to $300^{\circ} \mathrm{C}$ at $+30^{\circ} \mathrm{C} \mathrm{min}^{-1}\left(3 \mathrm{~min}\right.$ at $\left.300^{\circ} \mathrm{C}\right)$. The MS was operated in the electron impact mode $(70 \mathrm{eV})$ for $\mathrm{m} / \mathrm{z} 40$ to 450 . The various phenolic pyrolysis compounds were identified by comparison to the spectra of authentic compounds or to published spectra [42]. The identification and quantitative evaluation of the surface areas of the main pyrolysis-derived phenolics were performed using the freely-available Automatic Mass Spectral Deconvolution and Identification Software (AMDIS version 2.69).

In addition to the one-step pyrolysis method performed as described above, some analyses were carried out on initial PB1000 sample using a two-step thermal treatment. A first treatment at $200^{\circ} \mathrm{C}$ for $20 \mathrm{~s}$ was performed to simply volatilize low molecular weight aromatics, if any, followed by the pyrolysis step at $500^{\circ} \mathrm{C}$ for $15 \mathrm{~s}$ which induces the degradation of non-volatile compounds into a mixture of volatile pyrolysis adducts.

\section{ACKNOWLEDGEMENTS}

This project has received funding from the Bio Based Industries Joint Undertaking under the European Union's Horizon 2020 research and innovation program under grant agreement No 720303. We are grateful to Dr Richard Gosselink (FBR, Netherlands) for providing PB1000 and Dr David Sillam-Dussès for collecting the termite colony. We sincerely acknowledge the financial support of the 3BCAR Institut Carnot for the acquisition of the Py-GC/MS equipment. The IJPB research unit benefits from the support of the LabEx Saclay Plant Sciences-SPS (ANR-10-LABX-552 0040-SPS). 
1. Kamimura N, Takahashi K, Mori K, Araki T, Fujita M, Higuchi Y, Masai E: Bacterial catabolism of lignin-derived aromatics: New findings in a recent decade: Update on bacterial lignin catabolism. Environ Microbiol Rep 2017, 9(6):679-705.

2. Schutyser W, Renders T, Van den Bosch S, Koelewijn SF, Beckham GT, Sels BF: Chemicals from lignin: an interplay of lignocellulose fractionation, depolymerisation, and upgrading. Chem Soc Rev 2018, 47(3):852-908.

3. Xie S, Syrenne R, Sun S, Yuan JS: Exploration of Natural Biomass Utilization Systems (NBUS) for advanced biofuel--from systems biology to synthetic design. Curr Opin Biotechnol 2014, 27:195-203.

4. Tokuda G, Tsuboi Y, Kihara K, Saitou S, Moriya S, Lo N, Kikuchi J: Metabolomic profiling of 13Clabelled cellulose digestion in a lower termite: insights into gut symbiont function. Proc Biol Sci 2014, 281(1789):20140990.

5. Hongoh $Y$, Deevong $P$, Inoue $T$, Moriya $S$, Trakulnaleamsai $S$, Ohkuma $M$, Vongkaluang $C$, Noparatnaraporn N, Kudo T: Intra- and interspecific comparisons of bacterial diversity and community structure support coevolution of gut microbiota and termite host. Appl Environ Microbiol 2005, 71(11):6590-6599.

6. Ohkuma $M$, Brune $A$ : Diversity, structure, and evolution of the termite gut microbial community. . In., D.E. Bignell et al. (eds.), Biology of Termites: A Modern Synthesis edn; 2011.

7. Warnecke $F$, Luginbuhl $P$, Ivanova N, Ghassemian $M$, Richardson $T H$, Stege JT, Cayouette $M$, McHardy AC, Djordjevic G, Aboushadi $\mathrm{N}$ et al: Metagenomic and functional analysis of hindgut microbiota of a wood-feeding higher termite. Nature 2007, 450(7169):560-565.

8. Nakashima K, Watanabe H, Saitoh H, Tokuda G, Azuma JI: Dual cellulose-digesting system of the wood-feeding termite, Coptotermes formosanus Shiraki. Insect Biochem Mol 2002, 32 (7):777-784.

9. Brune $A$, Miambi E, Breznak JA: Roles of oxygen and the intestinal microflora in the metabolism of lignin-derived phenylpropanoids and other monoaromatic compounds by termites. Appl Environ Microbiol 1995, 61(7):2688-2695.

10. Harazono K, Yamashita N, Shinzato N, Watanabe $Y$, Fukatsu T, Kurane R: Isolation and characterization of aromatics-degrading microorganisms from the gut of the lower termite Coptotermes formosanus. Biosci Biotechnol Biochem 2003, 67(4):889-892.

11. Kuhnigk T, König $H$ : Degradation of dimeric lignin model compounds by aerobic bacteria isolated from the hindgut of xylophagous termites. J. Basic Microbiol 1997, 37(3):205-211.

12. Katsumata KS, Jin Z, Hori K, liyama K: Structural changes in lignin of tropical woods during digestion by termite, Cryptotermes brevis. J. Wood Sci. 2007, 53(5):419-426.

13. Griffiths B, Bracewell J, Robertson G, Bignell DE: Pyrolysis-mass spectrometry confirms enrichment of lignin in the faeces of a wood-feeding termite, Zootermopsis nevadensis and depletion of peptides in a soil-feeder, Cubitermes ugandensis. Soil Biol Biochem. 2013, 57:957-959.

14. Geib SM, Filley TR, Hatcher PG, Hoover K, Carlson JE, Jimenez-Gasco Mdel M, Nakagawa-Izumi A, Sleighter RL, Tien M: Lignin degradation in wood-feeding insects. Proc Natl Acad Sci U S A 2008, 105(35):12932-12937.

15. Ke J, Laskar D, Singh $D$, Chen S: In-situ lignocellulosic unlocking mechanism in termite for cellulose hydrolysis: critical lignin modification. Biotechnol. Biofuels 2011, 4:17-23.

16. Ke J, Laskar DD, Chen SJRe: Varied lignin disruption mechanisms for different biomass substrates in lower termite. Renew. Energy 2013, 50:1060-1064.

17. Ke J, Laskar DD, Chen S: Tetramethylammonium hydroxide (TMAH) thermochemolysis for probing in situ softwood lignin modification in each gut segment of the termite. J Agric Food Chem 2013, 61(6):1299-1308.

18. Brune A, Dietrich C: The Gut Microbiota of Termites: Digesting the Diversity in the Light of Ecology and Evolution. Annu Rev Microbiol 2015, 69:145-166. 
19. Brune A: Symbiotic digestion of lignocellulose in termite guts. Nat Rev Microbiol 2014, 12(3):168-180.

20. Ragauskas AJ, Beckham GT, Biddy MJ, Chandra R, Chen F, Davis MF, Davison BH, Dixon RA, Gilna $P$, Keller $M$ et al: Lignin valorization: improving lignin processing in the biorefinery. Science 2014, 344(6185):1246843.

21. Xu R, Zhang K, Liu P, Han H, Zhao S, Kakade A, Khan A, Du D, Li X: Lignin depolymerization and utilization by bacteria. Bioresour technol 2018.

22. Bugg TD, Ahmad $M$, Hardiman EM, Singh RJ: The emerging role for bacteria in lignin degradation and bio-product formation. Curr Opin Biotechnol 2011, 22(3):394-400.

23. Brune $A$, Emerson $D$, Breznak JA: The termite gut microflora as an oxygen sink: microelectrode determination of oxygen and $\mathrm{pH}$ gradients in guts of lower and higher termites. Appl. Environ. Microbiol. 1995, 61(7):2681-2687.

24. Brown ME, Chang MCJCoicb: Exploring bacterial lignin degradation. Curr Opin Chem Biol 2014, 19:1-7.

25. Diouf $M$, Roy $V$, Mora $P$, Frechault $S$, Lefebvre $T$, Hervé $V$, Rouland-Lefèvre $C$, Miambi $E$ : Profiling the succession of bacterial communities throughout the life stages of a higher termite Nasutitermes arborum (Termitidae, Nasutitermitinae) using 16S rRNA gene pyrosequencing. Plos One 2015, 10(10):e0140014.

26. Rossmassler K, Dietrich C, Thompson C, Mikaelyan A, Nonoh JO, Scheffrahn RH, Sillam-Dusses $D$, Brune $A$ : Metagenomic analysis of the microbiota in the highly compartmented hindguts of six wood- or soil-feeding higher termites. Microbiome 2015, 3:56.

27. Akalın MK, Karagöz S: Analytical pyrolysis of biomass using gas chromatography coupled to mass spectrometry. Trends Anal. Chem. 2014, 61:11-16.

28. Lupoi JS, Singh S, Parthasarathi R, Simmons BA, Henry RJ: Recent innovations in analytical methods for the qualitative and quantitative assessment of lignin. Renew. Sust. Energ. Rev. 2015, 49:871-906.

29. Constant S, Lancefield CS, Weckhuysen BM, Bruijnincx PC: Quantification and classification of carbonyls in industrial humins and lignins by 19F NMR. ACS Sustain. Chem. Eng. 2016, 5(1):965-972.

30. Santana AL, Maranhão CA, Santos JC, Cunha FM, Conceição GM, Bieber LW, Nascimento MS: Antitermitic activity of extractives from three Brazilian hardwoods against Nasutitermes corniger. Int Biodeterior Biodegradation 2010, 64(1):7-12.

31. He S, Ivanova N, Kirton E, Allgaier M, Bergin C, Scheffrahn RH, Kyrpides NC, Warnecke F, Tringe SG, Hugenholtz P: Comparative metagenomic and metatranscriptomic analysis of hindgut paunch microbiota in wood- and dung-feeding higher termites. PLoS One 2013, 8(4):e61126.

32. Boucias DG, Cai $Y$, Sun $Y$, Lietze VU, Sen R, Raychoudhury R, Scharf ME: The hindgut lumen prokaryotic microbiota of the termite Reticulitermes flavipes and its responses to dietary lignocellulose composition. Mol Ecol 2013, 22(7):1836-1853.

33. Raychoudhury R, Sen R, Cai Y, Sun Y, Lietze VU, Boucias DG, Scharf ME: Comparative metatranscriptomic signatures of wood and paper feeding in the gut of the termite Reticulitermes flavipes (Isoptera: Rhinotermitidae). Insect Mol Biol 2013, 22(2):155-171.

34. Su L, Liu YQ, Liu H, Wang Y, Li Y, Lin HM, Wang FQ, Song AD: Linking lignocellulosic dietary patterns with gut microbial Enterotypes of Tsaitermes ampliceps and comparison with Mironasutitermes shangchengensis. Genet Mol Res 2015, 14(4):13954-13967.

35. Schloss PD, Jenior ML, Koumpouras CC, Westcott SL, Highlander SK: Sequencing 16S rRNA gene fragments using the PacBio SMRT DNA sequencing system. PeerJ 2016, 4:e1869.

36. Mikaelyan A, Köhler T, Lampert N, Rohland J, Boga H, Meuser K, Brune A: Classifying the bacterial gut microbiota of termites and cockroaches: a curated phylogenetic reference database (DictDb). Syst App/ Microbiol 2015, 38(7):472-482.

37. Huang X-F, Bakker MG, Judd TM, Reardon KF, Vivanco JM: Variations in diversity and richness of gut bacterial communities of termites (Reticulitermes flavipes) fed with grassy and woody plant substrates. Microb. Ecol 2013, 65(3):531-536. 
38. Huang XF, Santhanam N, Badri DV, Hunter WJ, Manter DK, Decker SR, Vivanco JM, Reardon KF: Isolation and characterization of lignin-degrading bacteria from rainforest soils. Biotechnol Bioeng 2013, 110(6):1616-1626.

39. Zhu D, Zhang P, Xie C, Zhang W, Sun J, Qian W-J, Yang BJ: Biodegradation of alkaline lignin by Bacillus ligniniphilus L1. Biotechnol. Biofuels 2017, 10(1):44.

40. Narhi LO, Fulco AJ: J. Biol. Chem. 1986, 261(16):7160-7169.

41. Rashid GM, Taylor CR, Liu Y, Zhang X, Rea D, Fülöp V, Bugg TD: Identification of manganese superoxide dismutase from Sphingobacterium sp. T2 as a novel bacterial enzyme for lignin oxidation. ACS Chem. Biol. 2015, 10(10):2286-2294.

42. Ralph J, Hatfield RD: Pyrolysis-GC-MS characterization of forage materials. J. Agric. Food Chem. 1991, 39(8):1426-1437.

43. Tarmadi D, Tobimatsu $Y$, Yamamura M, Miyamoto T, Miyagawa $Y$, Umezawa T, Yoshimura T: NMR studies on lignocellulose deconstructions in the digestive system of the lower termite Coptotermes formosanus Shiraki. Sci Rep 2018, 8(1):1290.

44. Lapierre C: Application of new methods for the investigation of lignin structure. In: Forage cell wall structure and digestibility. American Society of Agronomy, 1993:133-166.

45. Dence CW: The determination of lignin. In: Methods in lignin chemistry. Springer; 1992: 3361.

46. Sipponen $\mathrm{MH}$, Lapierre $\mathrm{C}$, Mechin $\mathrm{V}$, Baumberger S: Isolation of structurally distinct lignincarbohydrate fractions from maize stem by sequential alkaline extractions and endoglucanase treatment. Bioresour Technol 2013, 133:522-528.

47. Lapierre C, Voxeur A, Karlen SD, Helm RF, Ralph J: Evaluation of Feruloylated and pCoumaroylated Arabinosyl Units in Grass Arabinoxylans by Acidolysis in Dioxane/Methanol. J. Agric. Food Chem. 2018, 66(21):5418-5424.

48. Barbosa P, Berry DL, Kary CS: Insect Histology: Pratical Laboratory Techniques. 2015:www.wiley.com/go/barbosa/insecthistology.

49. Magoč T, Salzberg SL: FLASH: fast length adjustment of short reads to improve genome assemblies. J. Bioinform. 2011, 27(21):2957-2963.

50. Martin M: Cutadapt removes adapter sequences from high-throughput sequencing reads. EMBnet j. 2011, 17(1):10-12.

51. Quast C, Pruesse E, Yilmaz P et al: The SILVA ribosomal RNA gene database project: improved data processing and web-based tools. Nucleic Acids Res. 2013, 41:590-D596

52. Rognes T, Flouri T, Nichols B, Quince C, Mahé F: VSEARCH: a versatile open source tool for metagenomics. PeerJ 2016, 4:e2584.

53. Mahé $F$, Rognes $T$, Quince $C$, de Vargas $C$, Dunthorn $M$ : Swarm: robust and fast clustering method for amplicon-based studies. PeerJ 2014, 2:e593.

54. Voxeur A, Soubigou-Taconnat L, Legée F, Sakai K, Antelme S, Durand-Tardif M, Lapierre C, Sibout $R$ Altered lignification in mur1-1 a mutant deficient in GDP-L-fucose synthesis with reduced RG-II cross linking. PloS One 2017, 12(9):e0184820.

\section{FIGURE AND TABLE TITLES}

Figure 1: Effect of diets in the survival rates of termites. The difference between the termites fed with

PB1000 and the control (birch wood diet) was statistically clear from day $2\left({ }^{*} p<0.05\right.$ according to

Fischer's PLSD post-doc test)

Figure 2: Changes in relative abundance of the major phyla in the guts of termites: A) directly collected

from their nest, B) fed with birch and C) fed with Protobind 1000 
Figure 3: Heatmap of the relative abundance of the major bacterial taxa in the guts of termites fed with birch wood (NEB); fed with Protobind 1000 (NEP) or directly collected from their nest (NEN). Classification is shown down to the family level

Figure 4: Partial pyrograms (total ion chromatograms) of A) Initial Protobind 1000 sample, B) termite guts containing digested Protobind 1000 sample, C) birch wood sample and D) termite guts containing birch wood sample.

Peak numbers correspond to the following compounds. 1: 4-hydroxy-5,6-dihydropyran-2-one; 2: 4methylphenol; 3: guaiacol; 4: 4-methylguaiacol; 5: 4-vinylphenol; 6: 4-ethylguaiacol; 7: 4-vinylguaiacol; 8: syringol; 9: vanillin; 10: 4-methylsyringol; 11: 4-allylguaiacaol; 12: acetoguaiacone; 13: 4ethylsyringol; 14: guaiacylacetone; 15: 4-vinylsyringol; 16: syringaldehyde; 17: 4-allylsyringol; 18: acetosyringone; 19: syringylacetone. $\mathrm{P} 1, \mathrm{P} 2$ and $\mathrm{P} 3$ peaks specific for termite gut samples are indicators of proteins and correspond to phenylacetonitrile (P1), indole (P2) and methylindole (P3). Peak 2 can also originate from protein tyrosine residues.

Additional file 1: GC/MS analyses (total ion chromatograms) of the low molecular weight phenolics released by two successive thermal treatments carried out on Protobind 1000 sample at A) $200^{\circ} \mathrm{C}$ (inducing the simple volatilization of soluble phenolics) and then at $\mathrm{B}$ ) $500^{\circ} \mathrm{C}$ (genuine pyrolysis).

Peak numbers correspond to the following compounds. 3: guaiacol; 4: 4-methylguaiacol; 5: 4vinylphenol; 6: 4-ethylguaiacol; 7: 4-vinylguaiacol; 8: syringol; 9: vanillin; 10: 4-methylsyringol; 11: 4allylguaiacol; 12: acetoguaiacone; 13: 4-ethylsyringol; 14: guaiacylacetone; 15: 4-vinylsyringol; 16 : syringaldehyde; 17: 4-allylsyringol; 18: acetosyringone; 19: syringylacetone.

\section{Declaration}

\section{- Ethics approval and consent to participate}

Experiments were performed with termites from the collection of the French Institute for Development (IRD) - Ile de France. Termites are not considered as endangered macrofauna. The termites used in the present study were obtained with the authorization. 
507

508

509

510

511

512

513

514

515

516

517

518

519

520

521

522

523

524

525

526

527

As corresponding author, I confirm that this manuscript has not been previously published and is not currently under consideration by any other journal. Additionally, all of the authors have approved the contents of this paper and have agreed to the BMC Biotechnology submission policies.

\section{- Availability of data and materials}

All data generated or analyzed during this study are included in this published article and its supplementary information files. The dataset of termite gut-bacterial community generated and analyzed during the current study is available in the NCBI Sequence Read Archive under the BioProject PRJNA550212 (https://www.ncbi.nlm.nih.gov/bioproject/?term=PRJNA550212).

\section{- Competing interests}

Not applicable

\section{- Funding}

This project has received funding from the Bio Based Industries Joint Undertaking under the European Union's Horizon 2020 research and innovation program under grant agreement No 720303

\section{- Authors' contributions}

MDJ performed the feeding-experiments and the molecular analysis of bacterial diversity; LC performed Py-GS/MS analysis; FP contributed in experimental design and in Py-GS/MS interpretation; SB participated in experimental design, data interpretation and manuscript editing; AR conceived feeding experiments and analysis of data; $\mathrm{CL}$ analyzed the Py-GS/MS data, performed analysis of monomeric phenols and the determination of volatile phenolic monomers released by pyrolysis and wrote the manuscript; MD and PM participated in experiment design of feeding experiments and discussion of the results. EM coordinated the whole study and wrote the manuscript. All authors 
528 suggested modifications to the draft, commented on several preliminary versions of the text, and

529 approved the final manuscript.

\section{- List of abbreviations}

531 NBUS: Natural Biomass Utilization Systems

532 Protobind 1000: PB 1000

533 Py-GC/MS: Pyrolysis Gas Chromatography Spectrometry

534 S/G: Syringyl-to-Guaiacyl ratio

535 KL: Klason Lignin

\section{- Acknowledgements}

537 This project has received funding from the Bio Based Industries Joint Undertaking under the European

538 Union's Horizon 2020 research and innovation program under grant agreement No 720303 . We are

539 grateful to Dr Richard Gosselink (FBR, Netherlands) for providing PB1000 and Dr David Sillam-Dussès

540 for providing the termite colony to IEES. We sincerely acknowledge the financial support of the 3BCAR

541 Institut Carnot for the acquisition of the Py-GC/MS equipment. The IJPB research unit benefits from

542 the support of the LabEx Saclay Plant Sciences-SPS (ANR-10-LABX-552 0040-SPS). 
Figures

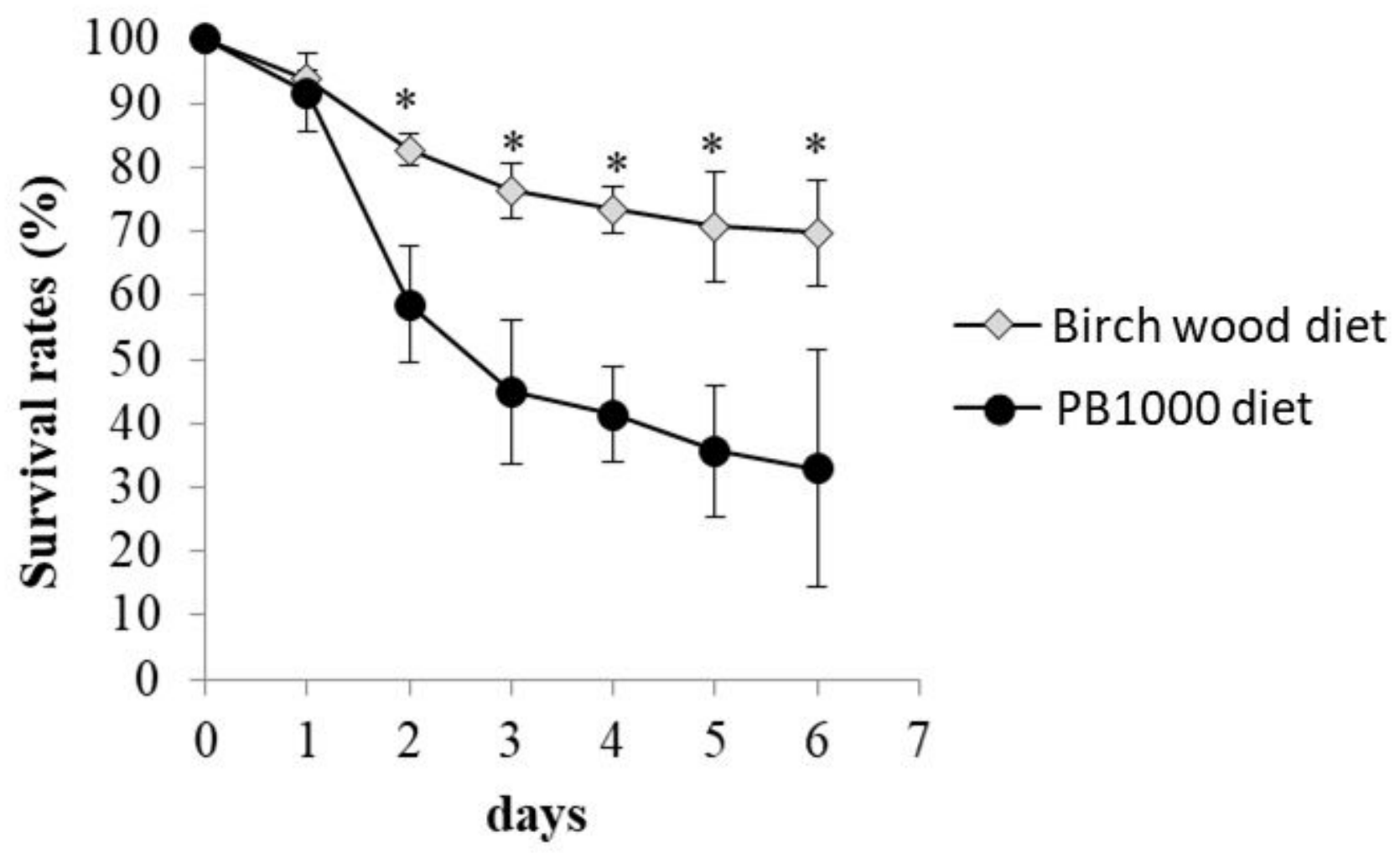

Figure 1

Effect of diets in the survival rates of termites. The difference between the termites fed with PB1000 and the control (birch wood diet) was statistically clear from day 2 ( ${ }^{*} \mathrm{p}<0.05$ according to Fischer's PLSD postdoc test) 


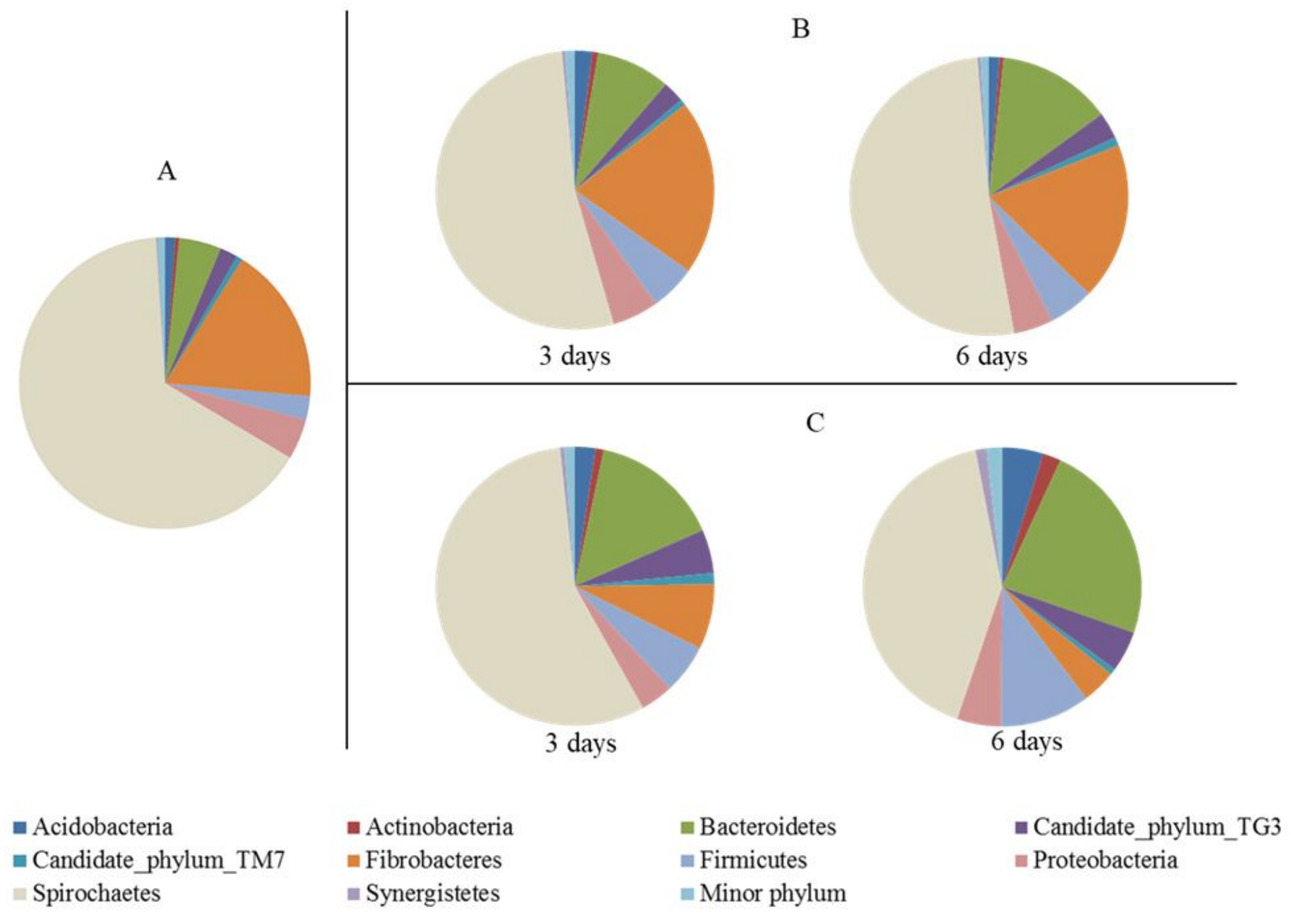

Figure 2

Changes in relative abundance of the major phyla in the guts of termites: A) directly collected from their nest, B) fed with birch and C) fed with Protobind 1000 


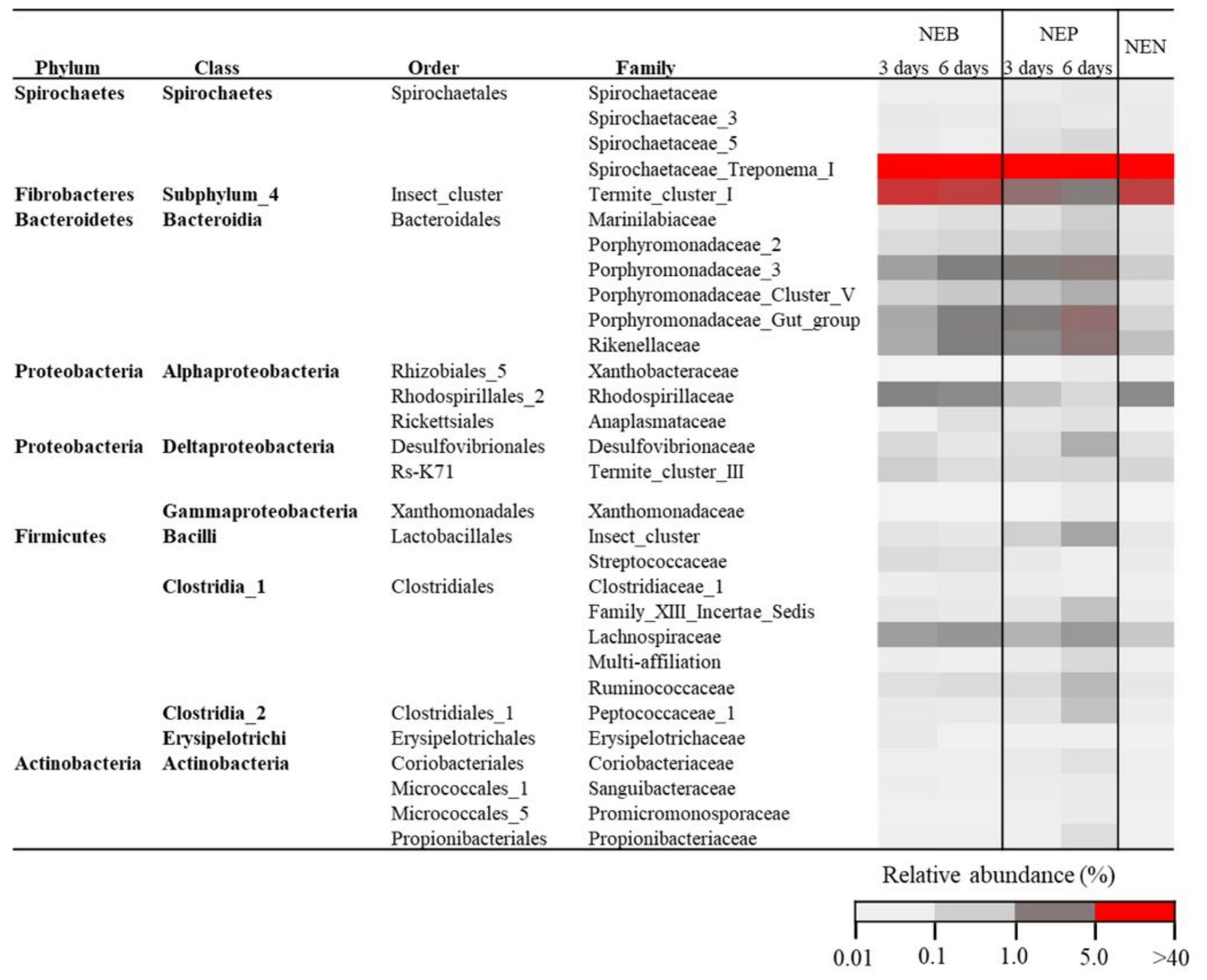

\section{Figure 3}




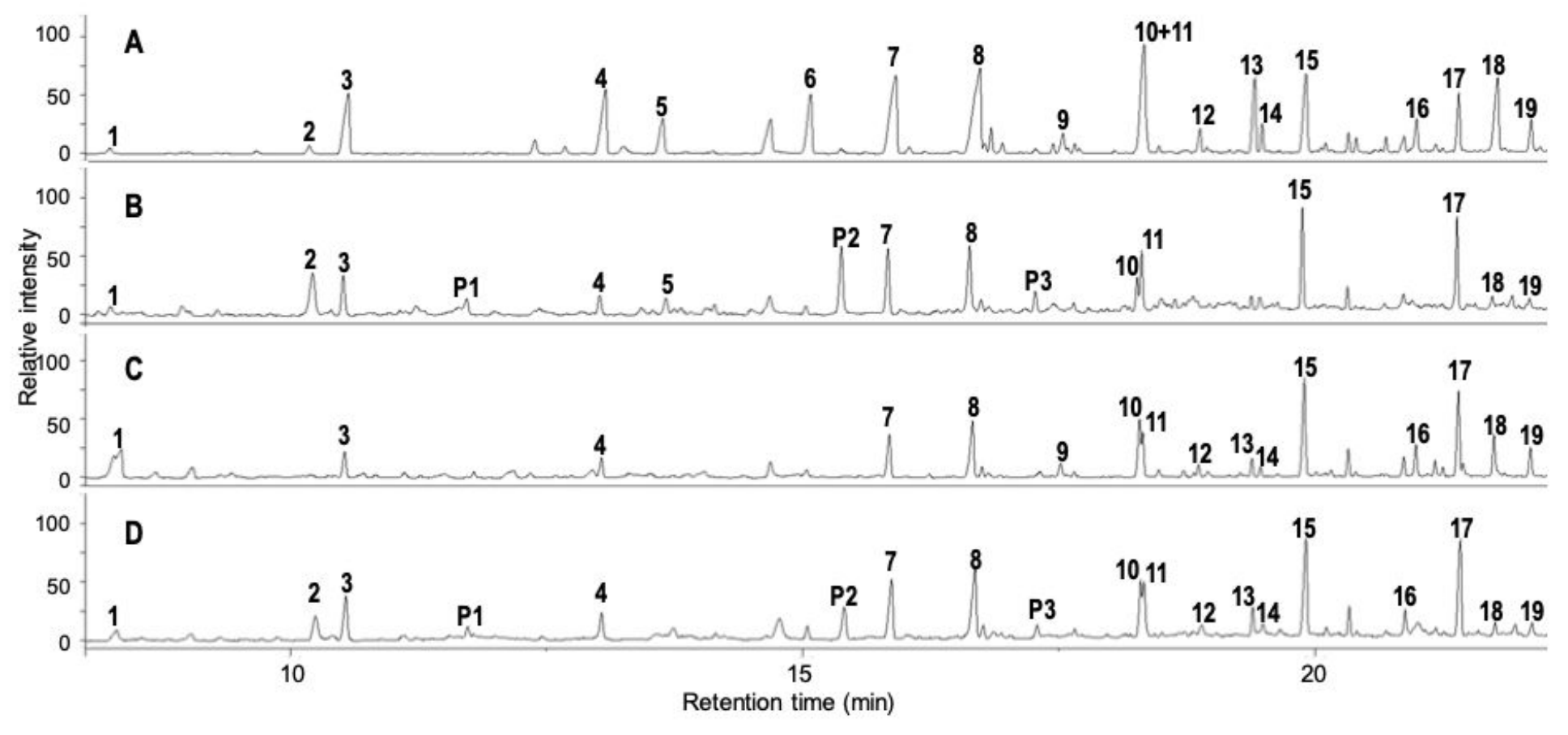

\section{Figure 4}

Partial pyrograms (total ion chromatograms) of A) Initial Protobind 1000 sample, B) termite guts containing digested Protobind 1000 sample, $C$ ) birch wood sample and D) termite guts containing birch wood sample. Peak numbers correspond to the following compounds. 1: 4-hydroxy-5,6-dihydropyran-2one; 2: 4- methylphenol; 3: guaiacol; 4: 4-methylguaiacol; 5: 4-vinylphenol; 6: 4-ethylguaiacol; 7: 4vinylguaiacol; 8: syringol; 9: vanillin; 10: 4-methylsyringol; 11: 4-allylguaiacaol; 12: acetoguaiacone; 13: 4ethylsyringol; 14: guaiacylacetone; 15: 4-vinylsyringol; 16: syringaldehyde; 17: 4-allylsyringol; 18: acetosyringone; 19: syringylacetone. P1, P2 and P3 peaks specific for termite gut samples are indicators of proteins and correspond to phenylacetonitrile (P1), indole (P2) and methylindole (P3). Peak 2 can also originate from protein tyrosine residues. 